(Aus dem physiologischen Laboratorium in Zürich.)

\title{
Untersuchungen zur Lehre von der electrischen Muskel- und Nervenreizung.
}

Von

\section{Hermann.}

\section{Ueber den Einfluss der absoluten Stromdichte auf die erregende Wirkung von Stromessehwankungen.}

Nach Versuchen von cand. med. Jacob Iselin aus Glarus und Theodor Wartmann aus St. Gallen, sowie vom Verfasser selbst.

Die zuerst von $\mathbf{d u}^{\mathrm{d}}$ Bois-Reymond ${ }^{1}$ ) aufgeworfene Frage, welchen Einfluss auf die erregende Wirkung einer Stromesschwankung die absolute Höhe der Stromdichte habe, auf welche sich die Schwankung superponirt, ist durch die bisherigen Untersuchungen nicht erledigt worden; ja für den Muskel existirt noch gar keine Untersuchung dieses Punctes. In meiner allgemeinen Nervenphysiologie ${ }^{2}$ ) habe ich entwickelt, welche Einfliusse aus dem Electrotonus des Bestandstroms za erwarten seien, und behufs Prüfung dieser Entwicklung habe ich die Herren cand. med. Is elin und Wartmann zu Versuchen aufgefordert, welche im Sommersemester 1882 ausgefuhrt wurden. Nach Beendigung derselben habe ich selber noch einmal alle Versuche wiederholt, mit Ergänzungen und kleinen Vervollkommnungen des Verfahrens.

1). Untersuchungen über thierische Electricität, I, p. 293; Abhandlungen der Berliner Akad. 1863, p. 137 (Ges. Abhandl. II, p. 204).

2) Handbuch der Physiologie, II, 1, p. 74 ff. und 83; hier sind auch die früheren Untersuchungen übersichtlich dargestellt. 


\section{Methode.}

Die Versuchsanordnung war die von Eckhard und von Pflüger angewandte, $d$. h. die secundäre Spirale eines Inductionsapparates war in den Kreis des constanten Stromes und des thierischen Theiles aufgenommen. Nach Pflüger's Vorgang war der constante Strom ein Zweigstrom der constanten Kette. Zur Abzweigung diente anfangs das du Bois'sche Rheochord, später, da dessen Schieber nicht immer ganz zuverlässig ist, ein Siemens'scher Stöpselrheostat von 0,1 bis 1000 Einheiten. Auch in den Hauptkreis der Kette war ein Stöpselrheostat (von 1 bis 10000 Einheiten) eingeschaltet.

Für die Versuche am Muskel (meist Sartorius, seltener Gastrocnemi us, beide völlig unversehrt) wurde die Richtung des constanten Stromes nicht verändert, sondern nur die des (Oeffnungs-) Inductionsstromes, mittels einer in seine Leitung eingeschalteten Wippe. Natürlich muss jedesmal für die betr. Richtung der minimal reizende Rollenabstand aufgesucht werden. Diese Arbeit wurde zur Hälfte dadurch gespart, dass für jede neue Stufe des Bestandstromes mit derjenigen Richtung des Reizstroms begonnen wurde, mit welcher bei der vorigen Stufe zuletzt gereizt war. Bei jeder Stufe wurde mehrmals abwechselnd mit und ohne Bestandstrom gereizt. Bei den Versuchen am Nerven musste auch die Richtung des Bestandstroms mittels einer in den Kettenkreis eingeschalteten Wippe gewechselt werden, so dass jedesmal vier Combinationen zur Anwendung kamen. Beim Nerven wurden stets unpolarisirbare Electroden verwendet, während beim Muskel die Ströme meist durch die Metalle direct zugeleitet wurden. Jedoch habe ich auch am Muskel eine Anzahl von Versuchsreihen mit unpolarisirbaren Electroden angestellt (Fadenzuleitung vom Thon zum Muskel), ohne dass die Resultate andere waren. In den Versuchen am Nerven wurden die beiden Electroden stets der untersten Strecke angelegt, um die Nähe der künstlichen Querschnitte am oberen and mittleren Theil des Nerven zu vermeiden (vgl. hierüber den Schlussparagraphen dieser Mittheilung). Für die Muskelversuche wurden stets curarisirte Frösche verwendet, obwohl in einigen Vergleichsversuchen ohne Curare die Resultate die gleichen waren. Schliesslich sei bemerkt, dass die Oeffnung des pri- 
Untersuchungen zur Lehre von d. electrischen Muskel- v. Nervenreizung. 3

mären Kreises mittels eines federnden Punctcontactes aus freior Hand geschah; die Zuckungshöhen erwiesen sich dabei hinreichend constant. Die Schliessungsinduction zeigte sich, da minimale Stromstärken verwandt wurden, stets ohne erregende Wirkung, wenn nicht der Bestandstrom solche bedingte; in diesem Falle wurde die betr. Zuckung nicht mit aufgeschrieben, ebensowenig: die Zuckungen bei Schliessung oder Oeffnung des Bestandstromes.

\section{Resultate.}

Das Hauptresultat lässt sich kurz dahin fassen, dass (wie ich schon im Handbuche voraus vermuthet habe) sowohl am Nerven als am Muskel die Wirkung eines gegebenen Inductionsstromes durch gleichgerichtete Bestandströme erhöht, durch entgegengesetzte herabgesetzt wird (bis zur Annullirung). Diese Wirkung beginnt schon bei so schwachen Bestandströmen, welche noch keine Schliessungszuckung geben, und erstreck:t sich bis zu so starken Strömen wie 1 Daniell beim Nerven und 2 Grove's beim Muskel ${ }^{1}$ ).

Bei den allerstärksten Strömen (Nerv von etwa 1 Grove, Muskel von etwa 2 Grove's ab) wird die erregende Wirkung des Inductionsstromes durch beide Richtungen des Bestandstroms vermindert und aufgehoben. Im Falle des gleichgerichteten Bestandstroms findet also bei einer gewissen hohen Stromstärke ein Uebergang von der erböhenden zur vermindernden Wirkung Statt. Am Nerven liegt dieser Wendepunkt für ab- und aufsteigende Bestandströme meist bei derselben Stromstärke; ist ein Unterschied vorhanden, so findet immer die Wendung für den aufsteigenden Strom bei geringerer Stromstärke Statt.

Was die Wirkung der allerschwächsten Ströme betrifft, so beginnt zuweilen das oben aufgestellte Grundgesetz schon bei den niedrigsten ibberhaupt wirksamen Stromstufen. In anderen Fällen aber treten hier Abweichungen ein. Sie bestehen meist darin,

1) Beides mit ganz entwickeltem Nebenschliessungswiderstand von 1000 Siem.-Einh. Indess ist in diesem Falle der Stromzweig im Muskel, resp. Nerv, ebenso stark, als wenn gar keine Nebenschliessung vorhanden wäre (vgl. hierüber J. Rosenthal, Electricitätslehre für Mediciner, 2. Auflage, p. 89; Hermann, Handbuch der Physiologie, II, 1, p. 31). 
dass die allerschwächsten entgegengesetzten Bestandströme erhöhend statt vermindernd wirken, und nicht selten auch die allerschwächsten gleichgerichteten Bestandströme vermindernd statt erhöhend. Diese Abweichungen kommen beim Muskel weit regelmässiger zur Beobachtung als beim Nerven, wo meist das Hauptgesetz gleich bei den schwächsten Strömen in die Erscheinung tritt; vermindernde Wirkung schwächster gleichgerichteter Ströme scheint beim Nerven nie vorzukommen.

Folgende Tabellen geben eine Uebersicht der beobachteten Erscheinungen.

Tabelle 1. Muskel.

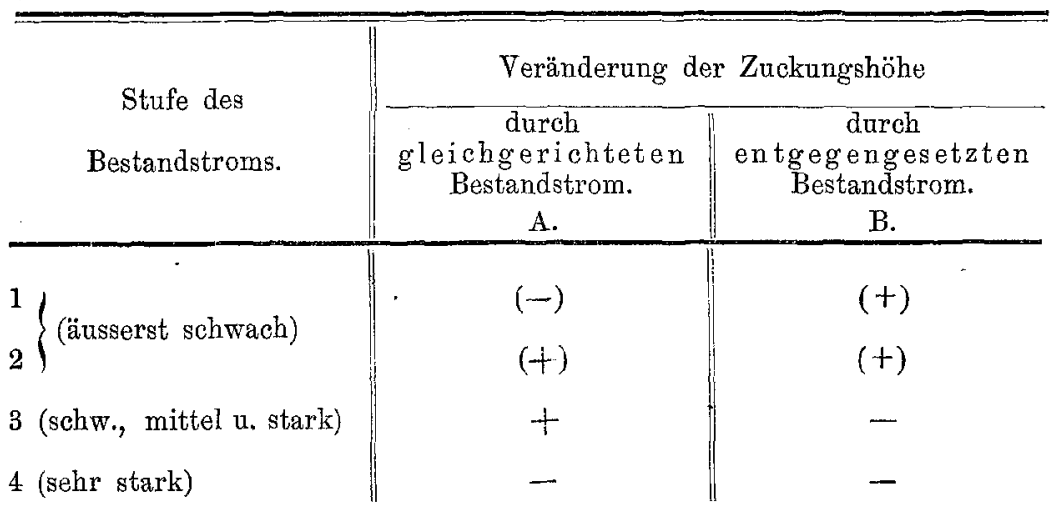

Tabelle 2. Nerv.

\begin{tabular}{|c|c|c|c|c|}
\hline \multirow[b]{2}{*}{$\begin{array}{c}\text { Stufe des } \\
\text { Bestandstroms. }\end{array}$} & $\begin{array}{r}\text { Verän } \\
\text { absteigende }\end{array}$ & $\begin{array}{l}\text { derung der Zuck } \\
\text { n Bestandstrom }\end{array}$ & $\begin{array}{l}\text { kungsgrösse dur } \\
\text { aufsteigende }\end{array}$ & $\begin{array}{l}\text { oh den } \\
\text { n Bestandstrom }\end{array}$ \\
\hline & $\begin{array}{l}\text { bei gleich- } \\
\text { gerichtetem } \\
\text { Inductions- } \\
\text { strom. } \\
\text { C. }\end{array}$ & $\begin{array}{c}\text { bei entgegen- } \\
\text { gesetztem } \\
\text { Inductions- } \\
\text { strom. } \\
\text { D. }\end{array}$ & $\begin{array}{l}\text { bei gleich- } \\
\text { gerichtetem } \\
\text { Inductions- } \\
\text { strom. } \\
\text { E. }\end{array}$ & $\begin{array}{c}\text { bei entgegen- } \\
\text { gesetztem } \\
\text { Inductions- } \\
\text { strom. } \\
\text { F. }\end{array}$ \\
\hline 1 (äusserst schwach) & 0 & $(-)$ & 0 & $(+)$ \\
\hline 2 (sehw., mittel u. stark) & + & - & + & - \\
\hline$\left.{ }_{4}^{3}\right\}$ (sehr stark) & $\begin{array}{l}(+) \\
-\end{array}$ & $\begin{array}{l}(-) \\
-\end{array}$ & $\begin{array}{l}(-) \\
-\end{array}$ & $(-)$ \\
\hline
\end{tabular}

Die eingeklammerten Zeichen bedeuten inconstantes Vorkommen der betr. Wirkungsstufe. 0 bedeutet: keine Veränderung. 
Untersuchungen zur Lehre von d. electrischen Muskel- u. Nervenreizung. 5

\section{Vergleichung der Resultate mit denjenigen früherer Autoren.}

Am Muskel sind bisher keine Versuche über unsere Frage angestellt worden. Fìr den Nerven existiren, wenn wir von Eckhard's nur sehr starke Ströme umfassenden Versuchen absehen, Versuche von Pflïger ${ }^{1}$ ) und 0 . Nasse ${ }^{2}$ ), welche jedoch nicht alle von uns untersuchten Combinationen betreffen.

Pflüger's Versuche betreffen nur gleichgerichtete Bestandströme, entsprechen also den Rubriken $\mathrm{C}$ nnd $\mathrm{E}$ der letzten Tabelle. Die Resultate stimmen für die von Pflüger untersuchten Fälle mit den unsrigen überein: Erhöhung bei schwachen, Verminderung bei starken Bestandströmen. Der Wechsel des Vorzeichens tritt nach Pflüger (S. 416) für auf- und absteigende Ströme bei der gleichen Stromstärke ein; in unsern Versuchen oft ebenso, oft aber (s. oben) bei aufsteigendem Strome friiher.

Nasse's Versuche entsprechen unseren Rubriken $\mathrm{C}$ und $\mathrm{F}$, mit dem Untersehiede, dass Nas se nicht Inductionsströme, sondern Schliessungen und Oeffnungen constanter Stromzuwachse benutzt hat; die Wegnahme eines gleichsinnigen Zuwachses kann aber, worauf ich schon im Handbuch (S. 77) hingewiesen habe, offenbar identificirt werden mit der Schliessung eines entgegengesetzten Stromes. Für positive Schwankungen wählte Nasse die absteigende, für negative die aufsteigende Stromrichtung, damit immer die Erregungsstelle auf die dem Muskel nähere Electrode fiele. Die Uebersicht über Nasse's Resultate wird dadurch erschwert, dass er den unnatürlichen und bedeutungslosen Quotienten $Q=\frac{e S}{u S}$ aufstellt, worin $u S$ den Bestandstrom, $e S$ den zur Erregung nöthigen minimalen Zuwachs darstellt. Wäre der Werth von $e S$ constant, d. h. hätte der Bestandstrom gar keinen Einfluss auf den erregenden Werth einer Schwankung, so würde $Q$ schon eine mit $\infty$ beginnende hyperbolische Curve darstellen; es kann aber keinen Nutzen haben, ohne genügenden Grund die gesuchte Grösse künstlich so zu gestalten, dass sie variabel wird, auch wenn sie an sich constant wäre. Dass ferner der Quotient $Q$ an

1) Untersuchungen über die Physiologie des Electrotonus, p. 392-428,

2) Dies Archiv, Bd. III, p. 476. 
sich kein Interesse bietet, zeigt sich besonders bei den negativen Stromesschwankungen. Hier kann nach $\mathrm{Nasse}$ die Untersuchung: erst bei demjenigen Werth des Bestandstroms beginnen, dessen Oeffnung zu einer Zuckung hinreicht: hier wäre also der Quotient $Q=1$. In dem einen Falle beginnt also die Untersuchung mit einem Werthe $Q=\infty$, im anderen mit $Q=1$; man sieht sofort, dass diese fundamentale Verschiedenheit zweier an sich gleichartiger Fälle nur auf der unglïcklichen Wahl des Werthes $Q$ beruht. Erwägt man nun aber, dass eine Verminderung des anfsteigenden Bestandstromes $A$ um die Grösse a physicalisch absolut gleichbedeutend ist mit der Hinzufügung eines absteigenden Stromes von der Grösse $a$, so sieht man, dass derjenige Bestandstrom, dessen Oeffnung eben noch Zuckung bewirkt, keine natürliche Grenze der Untersuchung bildet. Nennen wir diesen letzteren Bestandstrom $b$, so bildet den Gegenstand des Versuches die Wirkung der Schliessung des absteigenden Stromes $b$ bei aufsteigendem Bestandstrom $b$; warum sollten nun nicht auch bei schwächeren aufsteigenden Bestandströmen als $b$ diejenigen absteigenden Ströme gesucht werden können, deren Schliessung grade Zuckung macht?

Diese Ausstellungen an Nasse's Auffassung seiner Versuche stossen aber keineswegs den Werth der Versuche selbst um. Betrachten wir nur den Werth $e S$ (resp. a S), und setzen wir Erhöhung der Wirkung für Verminderung des erforderlichen Minimalreizes, so werden die Versuche mit den unsrigen einigermassen vergleichbar. Nasse erhielt hiernach für positive Schwankungen absteigender Ströme (entsprechend unserer Rubrik C) bei schwachen Bestandströmen erhöhte, bei stärkeren verminderte Wirkung, also dasselbe wie wir; dagegen für negative Schwankungen aufsteigender Ströme (entsprechend unserer Rubrik F) bei jeder Stärke des Bestandstroms (die niedersten Werthe desselben fehlen hier, wie erwähnt, in der Untersuchung) herabgesetzte Wirkung, also ebenfalls dasselbe was wir gefunden haben. Unsre Versuche weichen also in denjenigen Fällen, welche schon von frtiheren Autoren untersucht worden sind, nicht von deren Befunden ab, soweit sie vergleichbar sind. Neu ist unsern Versuchen, abgesehen von den Versuchen am Muskel und der vollständigeren Durchprïfung der Stromstärken, der Fall D, und wenn man auf den Gebrauch von Inductionsströmen Werth legt, auch der Fall F. Ausserdem aber konnte das eigentliche Gesetz des Einflusses der Bestandströme 
Untersuchungen zur Lehre von d. electrischen Muskel- u. Nervenreizung. 7

erst in den hier vorliegenden Versuchen hervortreten, weil sie zum ersten Male alle denkbaren Fälle umfassten.

\section{Schlüsse aus den Versuchen.}

Schon $d u$ Bois-Reymond weist daranf hin, dass in den Versuchen über den Einfluss von Bestandströmen auf die Wirkung von Stromesschwankungen zwei Dinge in Betracht kommen: 1. die electrotonische Wirkung des Bestandstroms auf die Erregbarkeit des Nerven; 2. der Einfluss der absoluten Ordinatenhöhen, zwischen denen eine Schwankung stattfindet, auf die erregende Wirkung der Schwankung. Wir wollen der Kürze halber den ersteren Einfluss als den des Electrotonus, den zweiten als den der „Verschiebung" bezeichnen, weil die Stromesschwankung durch den Bestandstrom ohne Veränderung •ihrer Grösse auf andere Ordinatenböhen verschoben wird. Zur Trennung beider Einflüsse führt $d u$ Bois-Reymond folgenden Versuchsplan an: vergleicht man dem Betrage nach den Einfluss des Bestandstroms auf die Wirkung electrischer und nicht electrischer Reize, so werden im ersteren Falle die Wirkung des Electrotonus und der Verschiebung vereinigt, im letzteren nur die des Electrotonus auftreten, so dass aus der Vergleichung der Beträge möglicherweise etwas über die verschiebende Wirkung sich ergeben würde. Die Ausführbarkeit dieses Planes wird aber von $d u$ Bo is-Reymond, wohl mit Recht, bezweifelt.

Pflüger hat bekanntlich die Resultate seiner einschlägigen Versuche rein electrotonisch zu erklären gesucht, indem er die Veränderungen des Reizerfolgs aus den Veränderungen der „totalen" Erregbarkeit der durchflossenen Strecke herleitete. Seitdem man aber weiss, dass (schwache) Inductionsströme nur an ihrer Cathode erregend wirken, kann diese Erklärung nicht mehr zugelassen werden, da sie voraussetzt, dass der Nerv durch den Inductionsstrom an jeder Stelle, die er durchläuft, erregt werde. Andrerseits hat $\mathrm{Nasse}$ bei der Erklärung seiner Resultate, wie mir scheint, zu sehr die verschiebende und zu wenig die electrotonische Wirkung des Bestandstroms beruieksiehtigt.

Die electrotonische Wirkung des Bestandstroms lässt sich leicht voraussagen, wie ich schon im Hanḑbuch entwickelt habe; bei positiven Schwankungen des Bestandstroms, oder, was hin- 
sichtlich der Erregung auf dasselbe hinausläuft, bei gleichgerichteten Inductionsströmen, fällt die Erregungsstelle auf eine bestehende Cathode, so dass erhöhte Wirkung zu erwarten ist; bei negativen Schwankungen oder entgegengesetzten Inductionsströmen fällt dagegen die Erregungsstelle auf eine Anode, so dass verminderte Wirkung zu erwarten ist. Modificirt können diese Einflüsse am Nerven werden durch die bekannten Wirkungen des Electrotonas auf das Leitungsvermögen (ich spreche absichtlich in den Ausdrticken der Pfl iiger'schen Theorie als der verbreiteteren, mache aber den Vorbehalt einer anderen, von mir aufgestellten Auffassung des Sachverhalts, welche in diesen Puncten zu denselben Resultaten führt); von den vier denkbaren Fällen trifft dies nur für einen $\mathrm{za}$, nämlich für aufsteigenden Bestand- und Zuwachsstrom, wo bei starken Strômen statt Erhöhung Verminderung eintreten müsste. Die electrotonischen Einhüssse, welche die Theorie erwarten lässt, wären also folgende:

Tabelle 3. (Folgerungen aus dem electrotonischen Gesetz)

\begin{tabular}{|c|c|c|c|c|}
\hline \multirow{3}{*}{$\begin{array}{c}\text { Intensität } \\
\text { des } \\
\text { Bestandstroms. }\end{array}$} & \multicolumn{4}{|c|}{ Veränderung der Wirkung } \\
\hline & \multicolumn{2}{|c|}{ Bestàndstrom $\downarrow$} & \multicolumn{2}{|c|}{ Bestandstrom $\uparrow$} \\
\hline & $\begin{array}{c}\text { Ind.-Str. } \downarrow \\
\text { (C) }\end{array}$ & $\begin{array}{c}\text { Ind.-Str. } \uparrow \\
\text { (D) }\end{array}$ & $\begin{array}{c}\text { Ind.-Str. } \uparrow \\
(\mathbf{E})\end{array}$ & $\begin{array}{c}\text { Ind.-Str. } \downarrow \\
(\mathrm{F})\end{array}$ \\
\hline Schwach u. mittelstark. & + & - & + & - \\
\hline Stark. & + & - & - & - \\
\hline
\end{tabular}

Abweichungen von diesem a priori construirten Verhalten können kaum anders als dureh die verschiebende Wirkung des Bestandstroms erklärt werden. Eine Vergleichung der Tabellen 1 und 2 mit 3 zeigt nun nur eine einzige entschiedene Abweichung; nämlich das Verhalten bei starken Bestandströmen im Falle A resp. C; hier wirken starke Bestandströme vermindernd und aufhebend auf die Erregung, obgleich sie electrotonisch erhöhend wirken müssten. Diese Thatsache, welche von allen Untersuchern constatirt ist, kann nicht von Widerstandsänderungen berrühren, denn bei starken Strömen ist der Widerstand des Rheochords oder des seine Stelle einnehmenden Rheostaten sehr gross, der des Kettenzweiges sehr klein; die hinzukommende Schliessung durch 
Untersuchungen zur Lehre von d. electrischen Muskel- u. Nervenreizung. 9

den Kettenzweig kann also die Intensität des Reizstromes nur verstärken, während es sich um Erklärung einer verminderten Wirkung handelt. Diese kann demnach nicht anders erklärt werden, als dadurch, dass starke Bestandströme die erregende Kraft einer minimalen Stromesschwankung ïberhaupt beseitigen. Ist dies auch nur für den Fall $\mathrm{C}$ wirklich erwiesen, so findet es doch wabrscheinlich auch in den Fällen D, E, F statt, wo die Beseitigung der Zuckung sehon electrotonisch erklärbar ist.

Diese Folgerung ist aber auch theoretisch ziemlich begreiflich; die erregende Wirkung einer Stromesschwankung beruht nämlich, wie wir jetzt wissen, nicht einfach auf der Veränderung der Stromdichte im Muskel oder Nerven, sondern auf der Veränderung einer Wirkung des Stromes, nämlich des Electrotonus oder der Polarisation. Minimale Dichtenänderungen werden aber um so schwieriger den Polarisationsbestand ändern, je höher derselbe bereits ist, und schliesslich ganz unwirksam sein. Wir haben also hier eine wabre Wirkung der "Versehiebung", welche möglicherweise ${ }^{1}$ ) schon bei schwächeren Bestandströmen auftritt, hier aber durch die besprochenen Wirkungen des Electrotonus verdeckt wird.

Vergleicht man das Verhalten der Rubriken $\mathrm{C}$ und $\mathrm{E}$; so zeigt sich zuweilen, wie schon oben hervorgehoben ist, der Uebergang von + zu - in der Rubrik E schon bei niedrigeren Stromstärken als in $\mathrm{C}$. Auch dies ist erklärbar; bei $\mathrm{C}$ ist dieser Uebergang lediglich Wirkung der Verschiebung, bei E aber Wirkung des Electrotonus und der Verschiebung; diese vereinte Wirkung wird schon bei schwächeren Strömen za Stande kommen können.

Bei den allerschwächsten Strömen zeigen die Versuche, wie man aus den Tabellen ersieht, zuweilen ein dem Grundgesetze entgegengesetztes Verhalten, nämlich Erhöhung der Erregung durch entgegengesefzten, Herabsetzung durch gleichgerichteten Bestandstrom. Am Nerven ist diese Umkehrung selten, und kommt nur in dem Falle $F$ zuweilen ror, nie in den Fällen C, D und $E$; d. h. die Wirkung des absteigenden Inductionsstromes wird durch die schwächsten aufsteigenden Bestandströme zuweilen erhöht statt vermindert. Gewöhnlicher ist die Unkehrung beim Muskel, wo sie bei sehr schwachen Bestandströmen fast stets mit der normalen

1) Bei schwachen Bestandströmen braucht sie nicht vorhanden zu sein, soweit die Polarisation der Stromdichte proportional wächst. 
Wirkung in Kampf tritt (s. Tabelle 1). Diese Umkehrung kann nicht von den. Veränderungen der Intensität des Reizstroms dureh die Schliessung des Bestandstromes herrïhren; denn erstens sind diese Veränderungen in allen Fällen dem Sinne nach Verstärkungen, weil zu der Schliessung des Kreises durch den Rheochordwiderstand $\left.{ }^{1}\right)$ noch eine Schliessung durch den geschlossenen Kettenzweig hinzukommt; zweitens sind diese Veränderungen verschwindend klein; denn bei den schwächsten Bestandströmen ist der Rheochordwiderstand äusserst klein (z. B. 0,1 Siem.-Einh.), der Widerstand des Kettenzweiges dagegen sehr gross (ausser der Kette selbst noch mindestens 100 S.-E.); neben der ersteren ist also die zweite Schliessung völlig wirkungslos ${ }^{2}$ ).

Es bliebe demnach nichts Anderes übrig, als die bei den allerschwächsten Strömen zuweilen beobachtete Umkehrung des Gesetzes wiederum aus der verschiebenden Wirkung des Bestandstromes berzuleiten. Ehe wir-aber diesen Schritt thun, ist es gut sich zu erinnern, dass die Reizung durch Inductionsströme zwar ein sehr bequemes, aber nicht das einfachste Versuchsverfahren ist; denn der Inductionsstrom besteht aus einem ansteigenden und einem absteigenden Antheil; der erstere muss nach der Theorie an der Cathode, der zweite an der Anode erregen; wenn nun auch die letztere Erregung für gewöhnlich vernachlässigt wird, so ist sie doch eine störende Complication, sobald es sich um die zartesten Verhältnisse handelt. Ich habe deshalb die Versuche iuber die Wirkung der allerschwächsten Bestandströme in einer besonderen Versuchsreihe mit Reizung durch Kettenströme wiederholt.

\section{Versuche mit Reizung durch Kettenströme.}

Das Verfahren bestand einfach darin, dass der lange Rheochorddraht $A B$ (ein sog. "langer Compensator") an seinen beiden Enden

1) So bezeichne ich den Widerstand der Nebenschliessung, jenseits welcher die Kette geschlossen und geöffnet wird, gleichgültig ob das verwendete Instrument ein Rheochord oder ein Stöpselrheostat ist.

2) Im angegebenen Falle z. B. verändert sich der Widerstand 0,1 durch Schliessung des Kettenzweiges in den Widerstand $\frac{1}{\frac{1}{0,1}+\frac{1}{100}}=0,0999$; diese Aenderung ist sehon an sich verschwindend, vollends aber neben dem im Kreise befindlichen Widerstand des Muskels oder Nerven. 
Untersuchungen zur Lehre von d. electrischen Muskel- u. Nervenreizung. 11

mit dem Muskel oder Nerven verbunden wird; der Drabt hat zwei Schieber S und $\mathrm{S}^{i}$; dem Stück AS wird der Strom der Bestandkette, dem Stück BS' der Strom der Reizkette zugeleitet; jede

Kette besteht aus 1 Da$A \mid$ Is $\quad$ Is $\mid B$ niell ${ }^{1}$ ), und in jeden Kettenkreis ist ein Siemens'scher Stöpselrheostat, ein Stromwender und ein Schlüssel eingeschaltet; der Schlüssel des Reizkreises hat Punctcontact und ist zu uniformen Schliessungen eingerichtet.

Nachdem durch Einschaltung geeigneter Widerstände in den Kreis der Reizkette und Verschieben des Schiebers $S^{\prime}$ diejenige Intensität des Reizstroms aufgesucht ist, welche schwache Schliessungszuckungen giebt, werden mittels AS Bestandströme eingeführt, welche mit den allerschwächsten Stufen beginnen. Mittels der beiden Wippen werden alle 4 Combinationen bergestellt: Reizstrom $\downarrow$, Bestandstrom $\downarrow$; Rstr. $\downarrow$, Bstr. $\uparrow$; Rstr. $\uparrow$, Bstr. $\downarrow$; Rstr. $\uparrow$, Bstr. $\uparrow$. Bei Versuchen am Muskel sind nur 2 dieser Combinationen nöthig. Die Versuche werden bei allen Stufen des Bestandstroms systematisch wiederholt. Doch werden starke Bestandströme hier nicht verwendet, weil die Kürze der Nebenschliessung AS (bis $500 \mathrm{~mm}$ ) dies verbietet, und der Versuchsplan sich nur auf die schwächeren Bestandströme erstreckt.

Das Resultat dieser Versuche ist nun überraschend einfach und constant. Ohne eine einzige Ausnahme ist, sowohl am Muskel wie am Nerven, die Schliessungszuckung des Reizstroms durch den Bestandstrom, sobald dieser die zur Wirkung überhaupt erforderliche Stärke erreicht hat, verstärkt, wenn beide Ströme gleichgerichtet sind, vermindert, wenn sie entgegengesetzte Richtung haben. Das Grundgesetz zeigt sich also hier bis zu den allerschwächsten Bestandströmen völlig ungestört. Das Versuchsverfahren gestattet eine unmittelbare Vergleichung der Intensitäten des Reiz- und des Bestandstroms, und da zeigt sich, dass die Wirkung der Bestandströme bei ungleich niedrigeren Intensitäten beginnt als die der Reizströme: so wird z. B. die Schliessungszuckung eines schwachen Stromes (Minimaireiz) noch deutlich verändert

1) Bei Versuchen am Muskel (Sartorius) reicht im Reizkreise ein Daniell nicht aus, sondern es mussten mindestens 2 Zinkkohlenelemente angewandt werden, neben welchen höchstens 20 S.-E. im Rheostat sein durften. 
durch einen $50 \mathrm{mal}$ schwächeren Bestandstrom. Oft tritt durch die Wirkung eines entgegengesetzten Bestandstromes, - der die Schliessungszuckung des Reizstroms vermindert oder unterdriuckt, eine Oeffuungszuckung des Reizstroms auf, welche sonst fehlt. Auch dies erklärt sich ohne Weiteres aus der oben angefiuhrten Grundbetrachtung.

\section{Fortsetzung der Folgerungen aus den Versuchen.}

Da die oben angeführten zuweilen vorkommenden Umkehrungen des Grundgesetzes bei sehr schwachen Strömen in den letzterwähnten Versuchen mit einer einwandfreieren Methode völlig wegbleiben, so müssen wir sie als eine vor der Hand nerklärte Unregelmässigkeit betrachten, welche durch den Gebrauch der Inductionsströme bedingt ist, und haben also keinen Grund eine Veränderung der essentiellen Reizwirkung dureh mässige Verschiebung der Stromesschwankung anzunehmen. Das vollständige Resultat der Untersuchung lautet also:

Wird eine erregende Stromesschwankung auf einen bestehenden constanten Strom superponirt, so zeigen sich von den sehwächsten bis zu starken Bestandströmen nur solche Veränderungen des Reizerfolges, welche sich aus der polarisirenden Wirkung des Bestandstroms erklären: es wird nämlich die Schliessungszuckung eines Kettenstromes und die Zuckung durch einen Inductionsstrom verstärkt durch gleichgerichtete, vermindert durch entgegengesetzte Bestandströme; die Oeffnungszuckung zeigt sich umgekehrt begünstigt durch entgegengesetzte Bestandströme. Von einem Einfluss der absoluten Ordinatenhöhe auf die erregende Wirkung einer Stromesschwankung ist bei schwachen und mittleren Ordinatenhöhen nichts nachzuweisen. Sehr starke Bestandströme wirken jedoch auf die erregende Wirkung von Minimalreizen durchweg essentiell vermindernd und unterdrückend.

Der letztere Umstand veranlasste mich noch za einer Untersuchung über den Einfuss starker Bestandströme auf die Wirkung maximaler Reize. Fs zeigte sich, dass diese letztere durch starke Bestandströme nicht unterdrückt wird, weder bei Inductionsströmen, noch bei Schwankungen von Kettenströmen. Im Allgemeinen bleibt die Zuckungsgrösse durch noch so starke Bestandströme unverändert. Beim Muskel zeigt sich zuweilen eine geringe 
Verstärkung der Zuckung durch beide Richtungen des Bestandstroms. Dieselbe erklärt sich leicht durch das Hinzukommen der Leitung durch den Kettenzweig des Bestandstroms, dessen Widerstand in diesem Falle klein ist, während der Rheochordwiderstand ganz entwickelt ist; der erregende Inductionsstrom wird also hier durch die Schliessung des Bestandstroms nicht unbeträchtlich verstärkt. Die Thatsache, dass selbst enorme Bestandströme (z. B. 9 Zinkkohlenelemente) die Erregung durch superponirte starke Stromesschwankungen nicht unterdrücken können, ist von grosser theoretischer Bedeutung, und wird in einem späteren Theil dieser Untersuchungen zur weiteren Erörtèrung kommen.

\section{Weitere Bemerkungen.}

Als die vorstehende Untersuchung: schon weit vorgeschritten, und die Hauptresultate bereits festgestellt waren, erschien eine Arbeit von Grützner ${ }^{1}$ ), welche, gelegentlich des Einflusses der Demarcationsströme des Nerven auf die Wirkung electrischer Reize, auch die von uns behandelte Frage berührt (S. 142-146). Grützner schliesst sich der von mir aufgestellten Betrachtung iiber die Wirkung der Bestandströme an, und findet die auf Grund derselben von mir gemachte Voraussagung in Gestalt des oben aufgestellten Grundgesetzes ebenfalls bestätigt. Jedoch hat Grïtzner nur (gelegentlich seiner Untersuchungen über den Einfluss der Demarcationsströme des Muskels und Nerven auf einige in nenerer Zeit beobachtete Erscheinungen) über einige hierher gehörige Puncte Versuche angestellt, da eine erschöpfende Behandlung der ganzen Frage nicht im Plane seiner Untersuchungen lag.

Ich kann bei diesem Anlass nicht unterlassen, meiner Freude Ausdruck zu geben, dass der von mir hervorgehobene und gegen unberechtigte Einwände vertheidigte Einfluss des Demareationsstromes auf die Erregbarkeit am künstlichen Querschnitt ${ }^{2}$ ) nun endlich allgemeiner erkannt und gewuirdigt wird ${ }^{3}$ ). Es ist aber nöthig, nochmals dringend vor dem Irrthum zu warnen, dass man

1) Dies Archiv, Bd. XXVIII, p. 130.

2) Vgl. dies Archiv, Bd. VII, p. 363, 1873; Bd. IX, p. 29, 1874; Vierteljahrschr. d. naturf. Ges. in Zürich, 1878, p. 36 (auch in Moleschott's Unters. z. Naturl., XII, p. 29); Handbuch der Physiologie, II, 1, p. 117, 180.

3) Vgl. Grützner, Breslauer ärztl. Zeitschr., 1881, Nr. 11, und aus- 
durch Compensation den Nervenstrom beseitigen könne. Ich wiederhole, was ich schon einmal einer unrichtigen Betrachtung Bernstein's gegenüber hervorheben musste ${ }^{1}$, dass der Nervenoder Muskelstrom niemals durch äussere Einwirkung compensirt werden kann, sondern nur seine nach aussen abgeleiteten $Z$ weigströme, während im Gegentheil die innere Abgleichung an Intensität zunehmen muss. Ist der Nervenstrom abgeleitet und der Strom compensirt, so verhält sich der Nerv genau so, als wäre ihm gar kein leitender Bogen angelegt; denn der Bogen ist jetzt stromlos und einen stromlosen Theil kann man aus jedem System entfernen, ohne dass an den übrigen Theilen des Systems sich irgend Etwas ändert²). Dass nun aber in einem unabgeleiteten Nerven oder Muskel der Demarcationsstrom vorhanden ist, ist an sich klar. Um jede Demareationsfläche herum gleichen sich die Demarcationsströme durch die indifferenten Hüllen und Zwischengewebe der Fasern ab, und zwar, worauf ich ebenfalls schon längst hingewiesen habe, mit einer in nächster Nähe wahrhaft ungeheuren Intensität, wegen der Kleinheit der Widerstände bei den microscopischen Dimensionen. Es ist also gar nicht zu bezweifeln, dass unmittelbar an einem künstlichen Querschnitt ein enorm starker Catelectrotonus herrscht, wahrscheinlich stärker als wir ihn jemals durch künstliche Durchströmung des Nerven hervorbringen können. Hieran wird durch Ableitung und Compensation des Demarcationsstroms Nichts geändert; die $\mathbf{A b}$ leitung wird die innere Abgleichung um einen verschwindend kleinen Betrag vermindern, die Compensation des abgeleiteten Zweiges aber, wie gesagt, den früheren Zustand wieder herstellen. Compensirt man also den Nervenstrom, so wird die innere Wir-

führlicher in der eben citirten Abhandlung in diesem Archiv; ferner Biedermann, Sitzungsber. d. Wiener Akad., 3. Abth., LXXXV, p. 144 u. 159; und Hering, ebendaselbst, p. 237, 248 f. und 247.

1) Vgl. Bernstein, Dies Archiv, Bd. VIII, p. 504, und meine Entgegnung, ebendaselbst, Bd. IX, p. 29.

2) Die Oeffuung in einem ableitenden Bogen, dessen Stromzweig compensirt ist, kann daher auch nun und nimmermehr eine Zuckung geben; dieser Versuch ist in der interessanten Arbeit von Biedermann, welche in der vorletzten Note genannt ist, öfters erwähnt, ohne dass aber das Resultąt als ein nach den Gesetzen der Stromvertheilung selbstverständliches hingestellt wäre; ich erlaube mir, diesen Punkt hier hervorzuheben. 
kung desselben nicht, wie Manche geglaubt haben, aufgehoben, sondern im Gegentheil um ein Minimum verstärkt. Man könnte nun auf die Idee kommen, etwa durch Uebercompensiren doch den Demarcationsstrom völlig zu beseitigen, dies ist aber ebenfalls durchaus unmöglich, da die Strömungscurven des Gegenstroms im Nerven ja ganz anderen Verlauf haben als die des Demareationsstroms, also eine innere Compensation nicht denkbar ist, um so weniger, je näher der eigentlichen electromotorischen Fläche; die enorm starken Stromfäden in deren nächster Umgebung werden durch noch so starke äussere Ströme nicht wesentlich modificirt werden, obwohl letztere eine besondere Polarisation im Nerven schaffen ${ }^{1}$ ).

Die starke electrotonische Wirkung des künstlichen Querschnitts des Gesammtnerven, und die schwächere der künstlichen Quersehnitte am Abgang der Oberschenkeläste ${ }^{2}$ ) macht es nothwendig, bei allen grundlegenden Versuchen die Nähe dieser Stellen sorgfältig zu vermeiden. In unsern Versuchen war dies doppelt nothwendig (vgl. oben S. 2); denn in der Nähe dieser Strecken hätte man ja einen fortwährenden starken Bestandstrom. Auch am Muskel musste aus gleichem Grunde jede Verletzung sorgfältig vermieden werden. Erscheinungen, welehe an Nerven gefunden sind, die mit Demarcationsflächen behaftet sind, und welche grossentheils grade von diesen herruihren, wie z. B. die Curve der Erregbarkeit längs des in gewöhnlicher Weise präparirten Ischiadicus, sollte man nicht als "Gesetze" bezeichnen, da es die Aufgabe der Physiologie ist, Fehlerquellen zu beseitigen, oder wenigstens ihren Einfluss auszusondern. Der Titel „dogmatische Darstellung meines Zuckungsgesetzes" in einer neueren Abhandlung v. Fleischl's ${ }^{3}$ ) wirkt daher fast komisch. „Sein Zuckungsgesetz", welches nichts Anderes ist als eine speciellere Zergliederung einer von mir zuerst beschriebenen Erscheinung ${ }^{4}$ ), ist doch offenbar lediglich ein Verhalten des n icht normalen, nämlich des durch künstliche Querschnitte modificirten Nerven. Oder glanbt

1) Vgl. hierzu die Bemerkungen in diesem Archiv, Bd. X, p. 237 f.

2) Ueber diese vgl. mein Handbuch der Physiologie, II, 1, p. 117, sowie die Arbeit von Grützner.

3) Arch. f. (Anat. u.) Physiol. 1882, p. 3.

4.) Dies Archiv, Bd. VII, p. 361. Ioh bedaure, den Leser noch einmal mit v. Fleischl's merkwürdigen Windungen in dieser an sich so ungemein 
v. Fleisehl wirklich, dass die Nervenfaser des Ischiadicus von Natur ,in drei Strecken zerfällt", und dass der Abgang der Oberschenkeläste für die durchgehenden Fussnerven ein physiologischer Punct ist? Man kann meiner Ansicht nach heute ïberhaupt nicht mehr von einem ,Zuckungsgesetz" sprechen ${ }^{1}$ ), seitdem Pfliuger den grössten Fortschritt dieses Gebietes begründet hat: die Summe unübersehbarer Erscheinungen, die man bis dahin Zuckungsgesetz nannte, unter ein wahres Gesetz unterzuordnen, nämlich die Erregung durch Entstehen negativer oder Verschwinden positiver Polarisation. Ein neues "Zuckungsgesetz" aufstellen heisst aber vollends, übersehbaren Fehlerquellen eine unverdiente Ehre anthun.

klaren Prioritätsfrage behelligen zu müssen (vgl. đies Arch., Bd.XXIV, p. 304). Nachdem man früher das sog. Zuckungsgesetz als für alle Theile des Nerven gleichmässig gültig betrachtet hatte, fand ich 1873 zufällig, dass aufsteigende Ströme am unteren, absteigende am oberen Nervenende regelmässig in ihrer Wirkung begünstigt sind, und entdeckte später 2 vereinzelte Beobachtungen, die sich diesem Verhalten unterordnen, in einer älteren Arbeit von $\mathrm{Helm-}$ holtz. v. Fleischl, der auf das von mir gefundene Verhalten, nachdem er es selbst ebenfalls beobachtet, weiter baute, sucht nun meine ibm wie es scheint unbequeme Priorität mit allen Mitteln aus der Welt zu schaffen. Alles was er in seiner neuesten Auslassung (in du Bois -Reymond's Arch., 1882 , p. 5, Anm.) zugiebt, ist, dass ich "etwas Hierhergehöriges gesehen (!) und dessen Wichtigkeit erkannt" habe. Aber was ich mitgetheilt habe, sei doch „falsch" (obwohl v. Fleischl einige Jahre später in seiner ersten Abhandlung genau dasselbe nochmals als neue Entdeckung publicirt hat), weil es eben nicht alle in der weiteren Specialuntersuchung gefundenen und in der zweiten Abhandlung erörterten Fälle umfasst. Zum Glück ist es noch nicht allgemein üblich, dem Entdecker einer neuen Thatsache dadurch zu lohnen, dass man ihn, nachdem man sie noch einmal entdeckt, zuerst gar nicht, und dann nach allerlei leeren Ausflüchten nur in Anmerkungen erwähnt, und auch das nur um ihn herabzusetzen.

Dass Grützner in seiner erwähnten Arbeit unterlassen hat (p. 136) mich vor v. Fleischi als den Auffinder. der Thatsache zu nennen, dass aufsteigende Ströme am unteren, absteigende am oberen Nervenende die wirksameren sind, beruht auf einem blossen Versehen; Herr Prof. Grützner hat mich ausdrücklich ermächtigt und ersucht, dies in seinem Namen zu erklären.

1) v. Fleischl wird mir hoffentlich nicht entgegenhalten, dass ich in meiner ersten Mittheilung, einem gewöhnlichen Sprachgebrauch folgend, sagte, es habe sich bei meiner rein zufälligen Beobachtung "das Gesetz" gezeigt, dass u. s. w. 